Research Paper

\title{
Thymopentin treatment of murine premature ovarian failure via attenuation of immune cell activity and promotion of the BMP4/Smad9 signalling pathway
}

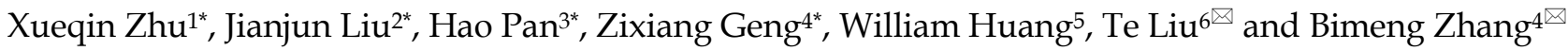 \\ 1. Geriatrics Department, Punan hospital of Shanghai, Shanghai 200031, China \\ 2. Trauma-Emergency \& Critical Care Medicine Center, Shanghai Fifth People's Hospital, Fudan University, Shanghai 200240, China. \\ 3. College of Pharmacy, Chongqing Medical University, Chongqing 400016, China. \\ 4. Department of Acupuncture, Shanghai General Hospital, Shanghai Jiao Tong University School of Medicine, Shanghai 200086, China. \\ 5. Hainan Zhonghe Pharmaceutical Co., Ltd, Hainan, China. \\ 6. Shanghai Geriatric Institute of Chinese Medicine, Shanghai University of Traditional Chinese Medicine, Shanghai 200031, China. \\ *These authors contributed equally to this work and shared the first authorship.
}

$\triangle$ Corresponding author: Prof. Bimeng Zhang, Department of Acupuncture, Shanghai General Hospital, Shanghai Jiao Tong University School of Medicine, 100 Haining Road, Shanghai 200080, China. Phone: 86-21-63240090; Fax: 86-21-63240090; E-Mail: Pjzhtiger08@aliyun.com. Prof. Te Liu, Shanghai Geriatric Institute of Chinese Medicine, Shanghai University of Traditional Chinese Medicine, 365 South Xiangyang Road, Shanghai, China, 200031, Phone: 86-21-64720010; Fax: 86-21-64720010; E-mail: liute1979@shutcm.edu.cn.

(C) The author(s). This is an open access article distributed under the terms of the Creative Commons Attribution License (https://creativecommons.org/licenses/by/4.0/). See http://ivyspring.com/terms for full terms and conditions.

Received: 2021.04.24; Accepted: 2021.08.06; Published: 2021.08.21

\begin{abstract}
Premature ovarian failure (POF) is a typical form of pathological aging with complex pathogenesis and no effective treatment. Meanwhile, recent studies have reported that a high-fat and high-sugar (HFHS) diet adversely affects ovarian function and ovum quality. Here, we investigated the therapeutic effect of thymopentin (TP-5) as a treatment for murine POF derived from HFHS and its target. Pathological examination and hormone assays confirmed that TP- 5 significantly improved murine POF symptoms. And, TP- 5 could reduce oxidative stress injury and blood lipids in the murine POF derived from HFHS. Flow cytometry and qPCR results suggested that TP-5 attenuated activation of CD3+ T cells and type I macrophages. RNA-Seq results indicated somedifferences in gene transcription between the TP- 5 intervention group and the control group. KEGG analysis indicated that the expression of genes involved in the mTOR signaling pathway was the most significantly different between the two groups. Additionally, compared with the control groups, the expression levels of interleukin, NFKB, and TNF families of genes were significantly downregulated in the POF+TP-5 group, whereas expression of the TGF $\beta / S m a d 9$ genes was significantly upregulated. Finally, immunofluorescence staining and qPCR confirmed that TP-5 promoted the polarization of Mø2 cells in the ovary by activating the expression of the BMP4/Smad9 signalling pathway. Thus, our study confirmed that TP-5 has a significant therapeutic effect on POF by upregulating BMP4/Smad9 signalling pathway so as to promote the balance and polarization of immune cell and reducing the release of inflammatory factors and reduce lipid oxidative stress injury.
\end{abstract}

Key words: Premature ovarian failure; high-fat and high-sugar (HFHS); thymopentin (TP-5); BMP4/Smad9 signalling pathway; immune cell activity

\section{Introduction}

Premature ovarian failure (POF) refers to a disorder in which women develop amenorrhea, infertility, low estrogen, and high gonadotropin levels, and lack of mature follicles before the age of 40 . This condition is one of the most common causes of female infertility [1-5]. The development of POF is closely related to the condition and quality of ovarian granulosa cells (OGCs) [1-5]. Aging and apoptosis of
OGCs are important causes of decreased ovarian reserve function [1-5]. It has been reported that inflammation can induce significant necrosis or apoptosis of OGCs, resulting in loss of their original function and ultimately inducing POF [1-5]; however, the specific mechanism by which this occurs is still unclear, and there are currently no effective treatment for POF. Previously, we demonstrated that oxidative 
stress-induced injury adversely affects OGC health [6]. Recent studies have reported that a high-fat and high-sugar (HFHS) diet increases the risk of obesity, tumor formation, and cardiovascular disease [7-9] and adversely affects ovarian function and ovum quality [10-12]. Meanwhile, we have reported that HFHS activated the Dab2ip/Ask1/p38-Mapk signaling pathway and promoted $\gamma \mathrm{H} 2 \mathrm{~A}$.X phosphorylation by inhibiting the expression of endogenous miR-146b-5p, which resulted in OGC aging and POF development [13]. Therefore, above evidences indicated that HFHS diet-induced obesity was closely related to the occurrence of POF.

Thymosin II is a single 49-amino acid polypeptide isolated from the thymus [14-18]. Thymopentin (TP-5), which is an active component of thymosin II, which is secreted by the thymus and has the same physiological functions [14-18]. TP-5 is composed of five amino acids: arginine, lysine, aspartic acid, valine, and tyrosine with the molecular formula $\mathrm{C}_{30} \mathrm{H}_{49} \mathrm{~N}_{9} \mathrm{O}_{9}$ and a molecular weight of 679.77 . Studies have shown that TP-5 has significant immunomodulatory effects [14-18]. It can promote the differentiation of $\mathrm{T}$ cells by increasing cAMP and intracellular GMP levels by binding to $\mathrm{T}$ cell-specific receptors, thereby inducing a series of intracellular reactions and regulating immune functions. In addition, TP-5 induces $\mathrm{T}$ cell differentiation, promoting the development, maturation, and activation of $\mathrm{T}$ lymphocytes subsets, and regulating the CD4/CD8 homeostasis of these subsets [14-18]. TP-5 has significant effects on the re-establishment of immune function after chemoradiotherapy in patients with malignant cancers, and in the immune system of elderly and immunodeficient individuals [14-18]. In addition, TP-5 has therapeutic effects on autoimmune diseases such as rheumatoid arthritis, lupus erythematosus, type II diabetes, and female climacteric syndrome [14-18]. However, the use of TP-5 to treat POF has not yet been reported.

Based on existing evidence, we investigated the therapeutic effects of TP-5 intervention in a murine POF derived from HFHS and explored the underlying mechanism from the perspective of immune cell activity and the release of inflammatory factors and lipid oxidative stress injury.

\section{Materials and methods}

\section{POF model and TP-5 treatment}

Thirty female C57BL/6 mice (aged 10 weeks) were purchased from the Experimental Animal Center of Shanghai University of Traditional Chinese Medicine (China). According to our previously established protocol $[5,7,13]$, the mice were divided into three groups: a group untreated with any reagents; a negative control group treated with saline daily by intraperitoneal injectionand HFHS (high-fat diet $(8 \mathrm{~g} / \mathrm{kg}$ bodyweight) and administered $200 \mu \mathrm{L}$ of $30 \%$ high fructose syrup once a day via gavage); an experimental group treated with $5 \mathrm{mg} / \mathrm{kg}$ TP-5 (Hainan Zhonghe Pharmaceutical Co., Ltd, Hainan, China)daily by intraperitoneal injection and HFHS (high-fat diet ( $8 \mathrm{~g} / \mathrm{kg}$ bodyweight) and administered $200 \mu \mathrm{L}$ of $30 \%$ high fructose syrup once a day via gavage). Following treatment, experiments using the animal model were conducted within 2 months. The mice were anesthetized and euthanized. Ketamine $(10 \mathrm{mg} / \mathrm{mL}) /$ Xylazine $(10 \mathrm{mg} / \mathrm{mL})$ was used for mouse anesthesia and administered $10 \mu \mathrm{L} / \mathrm{g}$ by intraperitoneal injection. All the animal experiments were conducted in accordance with the guidelines of the National Institutes for Health for the care and use of laboratory animals. The study protocol was also approved by the Committee on the Use of Live Animals in Teaching and Research, Experimental Animal Center of Shanghai University of Traditional Chinese Medicine, Shanghai, China (No. Shage20180001884).

\section{Hematoxylin and eosin (HE) staining}

According to a previously reported method [4], tissues were fixed in $4 \%$ paraformaldehyde at room temperature for $12 \mathrm{~h}$. Frozen tissue sections were prepared (thickness approximately $5 \mu \mathrm{m}$ ) and fixed in 95\% anhydrous ethanol for $2 \mathrm{~min}$. Sections were then stained in hematoxylin for $5 \mathrm{~min}$, and differentiated in differentiation solution for $2 \mathrm{~min}$. Sections were immersed in weak ammonia solution for $3 \mathrm{~min}$, washed with deionized water for $5 \mathrm{~min}$, and then stained with eosin for $5 \mathrm{~min}$. After washing with deionized water for $5 \mathrm{~min}$, tissue sections were immersed in $70 \%, 80 \%$, and $90 \%$ alcohol solution once for $1 \mathrm{~min}$, washed with anhydrous ethanol twice (1 min per wash), cleared in xylene twice $(1 \mathrm{~min}$ per wash), and mounted using neutral balsam. These reagents and materials were all purchased from Beyotime Biotechnology Co., Ltd., (Zhejiang, China).

\section{Immunofluorescence staining and flow cytometry}

According to previously reported methods [5], cell samples were fixed in $1 \mathrm{ml} 4 \%$ paraformaldehyde (Sigma-Aldrich) at room temperature for $30 \mathrm{~min}$ and blocked in blocking solution (Beyotime Biotechnology, Hangzhou, China) at $37^{\circ} \mathrm{C}$ for $60 \mathrm{~min}$. The blocking solution was discarded, and the cells were washed three times ( $5 \mathrm{~min}$ per wash) with an immunohistochemistry washing solution (Beyotime Biotechnology) at room temperature. Samples were 
then incubated at $37{ }^{\circ} \mathrm{C}$ for $45 \mathrm{~min}$ with primary detection antibodies (anti-mouse CD3 monoclonal antibody (mAb) (17A2), PE (Cat \#12-0032-82), anti-mouse CD4 mAb (45-0042-82), PerCP-Cyanine5.5 (Cat \#45-0042-82), anti-mouse CD8a mAb (53-6.7), PerCP-Cyanine5.5 (Cat \#45-0081-82), anti-mouse F4/80 mAb (BM8), Alexa Fluor 488 (Cat \#53-4801-82), anti-mouse CD68 mAb (FA-11), PE, (Cat \#12-0681-82), anti-mouse CD206 (MMR) mAb (MR6F3), PE (Cat \#12-2061-82); all from eBioscience, MA, USA; rabbit anti-mouse TGF- $\beta$ antibody (Cat \#3711), rabbit anti-mouse BMP4 (6B7) antibody (Cat \#4680), Smad 1/5/9 Antibody Sampler Kit (Cat \#12656), all from Cell Signaling Technology, MA, USA and Abcam, MA, USA). The antibodies were discarded, and the cells were washed three times (5 min per wash) with the immunohistochemistry washing solution (Beyotime Biotechnology) at room temperature. Samples were then incubated at $37^{\circ} \mathrm{C}$ for $45 \mathrm{~min}$ with secondary detection antibodies (goat anti-rabbit IgG H\&L (Alexa Fluor® 647) (ab150083); Abcam, MA, USA). The antibodies were discarded, and the cells were washed three times ( 5 min per wash) with the immunohistochemistry washing solution (Beyotime Biotechnology) at room temperature. Finally, the cells were mounted in immunofluorescence mounting fluid (Sigma-Aldrich). The cells were then detected using a flow cytometer (Cytomics FC500, BECKMAN).

\section{Establishment of cDNA sequencing libraries and high-throughput RNA-Seq}

The following analysis was conducted by Kang Chen Bio-tech (Shanghai, China). According to their experimental procedures, a random fragment sequencing library was constructed using a SOLiD Whole Transcriptome Analysis Kit (Life technologies, MA, USA). Nucleic acid cleaving reagents were added, and the mRNA was randomly disrupted into short segments in a shaking incubator. First-strand cDNA was reverse-transcribed using the fragmented mRNA as the template. Second-strand cDNA was synthesized using a second-strand DNA synthesis reaction system consisting of DNA polymerase I, dNTPs and RNase $\mathrm{H}$ (Sigma-Aldrich). The synthesized DNA was purified and recovered using a DNA purification kit. An adenine (A) base was added to the $3^{\prime}$ end of the cDNA, followed by ligation to the adapter, to complete the blunt end repair reaction. Subsequently, DNA fragment size selection was performed. Finally, the cDNA was used for PCR amplification to obtain a sequencing library. The quality of the constructed library was evaluated using an Agilent 2100 Bioanalyzer and the ABI StepOnePlus Real-Time PCR System and was subjected to high- throughput sequencing using an Illumina HiSeq TM 2000 Sequencer after passing quality control.

\section{ELISA}

The plasma levels of mouse estradiol $\left(E_{2}\right)$ were determined using an ELISA kit (Westang Bio, Shanghai, China) according to the manufacturer's instructions. Briefly, $100 \mu \mathrm{l}$ of mouse $\mathrm{E}_{2}$ standardized to $8,000,4,000,2,000,1,000,500,250$ and $125 \mathrm{pg} / \mathrm{mL}$ or diluted mouse plasma were added to anti- $\mathrm{E}_{2}$ antibody-precoated microwells and incubated for 60 min. After three washing steps, HRP-conjugated detection antibodies were added, followed by the substrate solution. The absorbance was measured at $450 \mathrm{~nm}$.

The plasma levels of mouse IL-1 $\beta$, IL-6, IL-22, TNFa and IFN $\gamma$ were determined using ELISA kits (Westang Bio, Shanghai, China) according to the manufacturer's instructions. Briefly, $100 \mu \mathrm{l}$ of mouse IL-1 $\beta$, IL-6, or TNFa standardized to 1,000, 500, 250, $125,60,30,15,0 \mathrm{ng} / \mathrm{ml}$ or diluted mouse plasma were added to microwells pre-coated with antibodies for the detection of the listed cytokines and incubated for $60 \mathrm{~min}$. In addition, $100 \mu \mathrm{l}$ of mouse IL-22, or IFNY standardized to $1,000,500,250,125,60,30,15,0 \mathrm{pg} / \mathrm{ml}$ or diluted mouse plasma were added to microwells pre-coated with antibodies for the detection of the listed cytokines and incubated for $60 \mathrm{~min}$. After three washing steps, HRP-conjugated detection antibodies were added, followed by the substrate solution. The absorbance was measured at $450 \mathrm{~nm}$.

\section{ATP assay}

The ATP assay was performed using an enhanced ATP test kit (Beyotime), according to the manufacturer's instructions. For the ATP assay, $1 \times 10^{5}$ cells/mL were lysed thoroughly with $200 \mu \mathrm{L}$ of sample lysate, centrifuged at $12000 \mathrm{~g}$ for $5 \mathrm{~min}$ at $4{ }^{\circ} \mathrm{C}$. The supernatant was carefully collected. To prepare the ATP standard curve, the ATP standard was adjusted with a dilution buffer to final concentrations of $0.01,0.03,0.1,0.3,1,3$, and $10 \mu \mathrm{M}$, and this curve was used as a reference to determine the value of the sample. Working solutions for ATP detection were freshly made in accordance with the kit's requirements. For the assay, $100 \mu \mathrm{L}$ of working solution was added to the test and sample wells at the same time, and the plates were incubated at room temperature for $5 \mathrm{~min}$. Then, $20 \mu \mathrm{L}$ of sample or standard was added to the wells at the same time, quickly mixed, incubated at room temperature for $5 \mathrm{~s}$, and the RLU value was measured on a luminometer.

\section{SOD assay}

According to the instructions of the SOD activity test kit (Beyotime), briefly, $1 \times 10^{5} / \mathrm{mL}$ of cells were 
lysed thoroughly using with $200 \mu \mathrm{L}$ of sample lysate, centrifuged at $12000 \mathrm{~g}$ for $5 \mathrm{~min}$ at $4{ }^{\circ} \mathrm{C}$, and the supernatant was collected. Then, fresh WST-8 enzyme working solution was prepared by mixing $151 \mu \mathrm{L}$ of SOD detection buffer with $8 \mu \mathrm{L}$ of WST-8 and $1 \mu \mathrm{L}$ enzyme solution. At the same time, the SOD standard was diluted to $100,50,20,10,5,2,1 \mathrm{U} / \mathrm{mL}$ to obtain a gradient. Then, $20 \mu \mathrm{L}$ of the cell lysis supernatant or standard solution wasadded to $160 \mu \mathrm{L}$ of fresh WST-8 enzyme working solution and $20 \mu \mathrm{L}$ reaction starting solution, mixed well, and incubated at $37^{\circ} \mathrm{C}$ for 30 min. Absorbance was measured at $450 \mathrm{~nm}$.

\section{RNA extraction and qPCR}

Total RNA was extracted from each group of cells in accordance with the instructions of Trizol Reagent (Invitrogen) and treated with Dnase I (Sigma-Aldrich) to remove residual genomic DNA. cDNA synthesis was carried out using ReverTra Ace-a First-Strand cDNA Synthesis Kit (Toyobo). qPCR analysis was conducted using a RealPlex4 real-time PCR detection system (Eppendorf Co. Ltd., Germany), with SYBR Green real-time PCR Master Mix (Toyobo) as a fluorescent dye for nucleic acid amplification. The following reaction conditions were used for 40 cycles: denaturation at $95{ }^{\circ} \mathrm{C}$ for $15 \mathrm{~s}$, annealing at $58{ }^{\circ} \mathrm{C}$ for $45 \mathrm{~s}$, andelongationat $72{ }^{\circ} \mathrm{C}$ for $42 \mathrm{~s}$. For each sample, the maker gene $\mathrm{Ct}$ values were normalized with the following formula: $\Delta \mathrm{Ct}$ $=\mathrm{Ct} \_$genes $-\mathrm{Ct} \_18 \mathrm{sRNA}$, and $\Delta \Delta \mathrm{Ct}=\Delta \mathrm{Ct} \_$all_groups$\Delta$ Ct_blankcontrol_group. The mRNA levels were calibrated according to the $18 \mathrm{~S}$ rRNA levels.

\section{Western blot}

Briefly, total protein was extracted from cells in each group. Protein content was determined by using the BCA assay (Pierce Biotechnology, Inc., Rockford, IL, USA). Briefly, $20 \mu \mathrm{g}$ protein samples were electrophoresed on 12\% SDS-PAGE. These parated proteins were transferred to a polyvinylidene difluoride (PVDF) membrane (Millipore, Billerica, MA, USA) for $45 \mathrm{~min}$ at $37^{\circ} \mathrm{C}$ after the blocking and membrane washing 4 times with TBST for 1 mineach time. Each membrane was washed and incubated with the secondary antibodies for $45 \mathrm{~min}$. Immunoreactivity was visualized by performing an enhanced chemiluminescence (ECL) assay using an ECL kit from Perkin-Elmer LifeScience (Norwalk, USA).

\section{Statistical analysis}

Each experiment was performed as least three times, and data represent the mean \pm SD where applicable. Differences were evaluated using Student's $t$-test. $P<0.05$ was considered to indicate statistical significance. In addition, about the follicular count method, under the microscope low power field of vision, the total number of normal follicles (including primordial follicles, secondary follicles, sinus follicles, etc.) and atresia follicles in a complete ovarian tissue were calculated respectively. Then, according to the formula (total number of atresia follicles/total number of normal follicles) $\times 100 \%$, the proportion of atresia follicles can be obtained.

\section{Results}

\section{TP-5 significantly improveslipidoxidative stress injury and POF symptoms}

Histopathological examination of ovarian tissue from each group showed few follicles in the ovarian tissue of the POF+Saline (HFHS+Saline) mice group, whereas multiple mature oocytes and follicular tissues were found in the ovaries of the normal (Untreated) group (Figure 1A). In TP-5-treated mice (HFHS+TP-5), the phenomenon of atretic follicles in ovarian tissue was significantly improved. Follicle counting indicated that the proportion of atretic follicles in the ovaries from the POF group was significantly higher than that from the Untreated group, whereas the proportion of atretic follicles in the ovaries from the TP-5 treated group was significantly lower than that from the POF group (Figure 1A, B). In addition, the weights of the POF mouse ovaries were significantly lower than those of the untreated group, whereas the weights of the ovaries of TP-5-treated mice were significantly higher than those of the POF group (Figure 1C). Peripheral blood $E_{2}$ levels were significantly lower in POF mice than those in the untreated group, whereas peripheral blood $E_{2}$ levels were significantly higher in the TP-5-treated group than those in the POF group (Figure 1D). Meanwhile, pathological evaluation showed that HFHS intervention in mice resulted in an increase in monocytes in the spleen and thymus tissues, which was alleviated by TP- 5 intervention (Figure 1E, F). Besides, compared with ovarian tissues in murine $\mathrm{POF}+$ Saline group, $\mathrm{CAT}, \mathrm{ATP}$ contents and SOD activity were higher and LPO, MDA, Superoxide anion radical contents were significantly lower in the TP-5-treated groups (Figure 2A). And, the levels of high density lipoprotein (HDL) in peripheral blood from TP-5 treated mice was significantly higher than it in $\mathrm{POF}+$ Saline group (Figure 2B). But, the levels of lowdensity lipoprotein (LDL) and the ratio of total cholesterol (TC) to HDL in peripheral blood from TP-5 treated mice were significantly lower than them in POF+Saline group (Figure 2B).These experimental results suggested that TP-5 significantly improved POF symptoms and HFHS mediated lipid oxidative stress injury. 

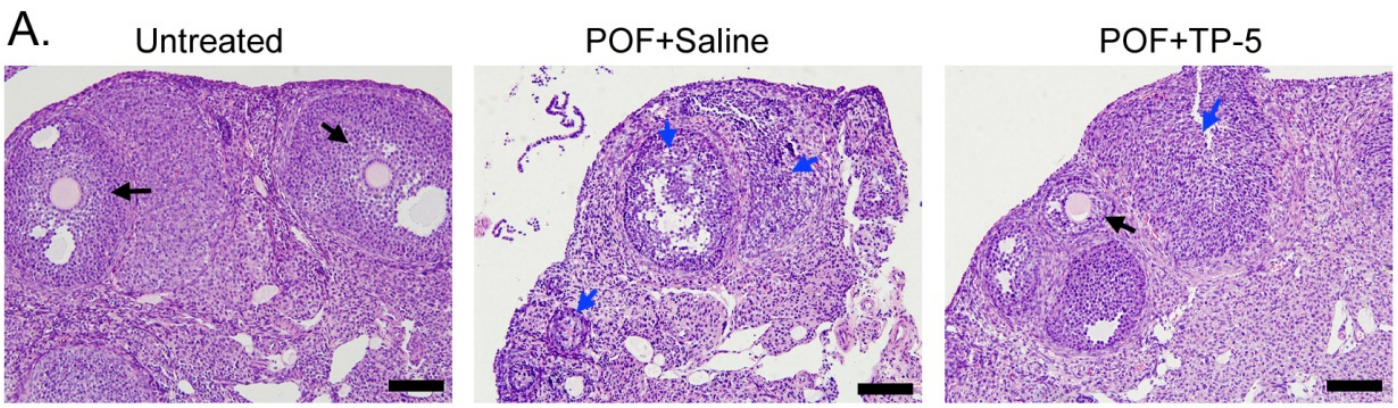

B.

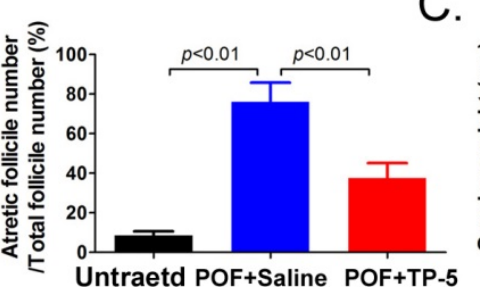

E.

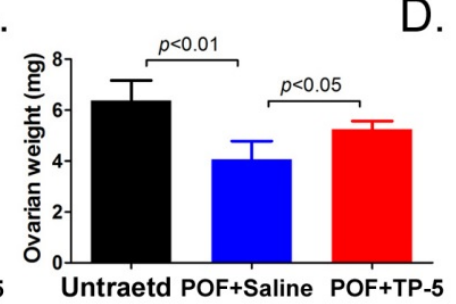

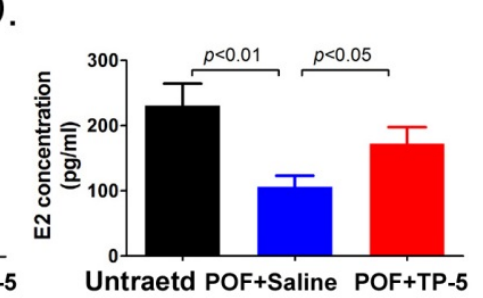

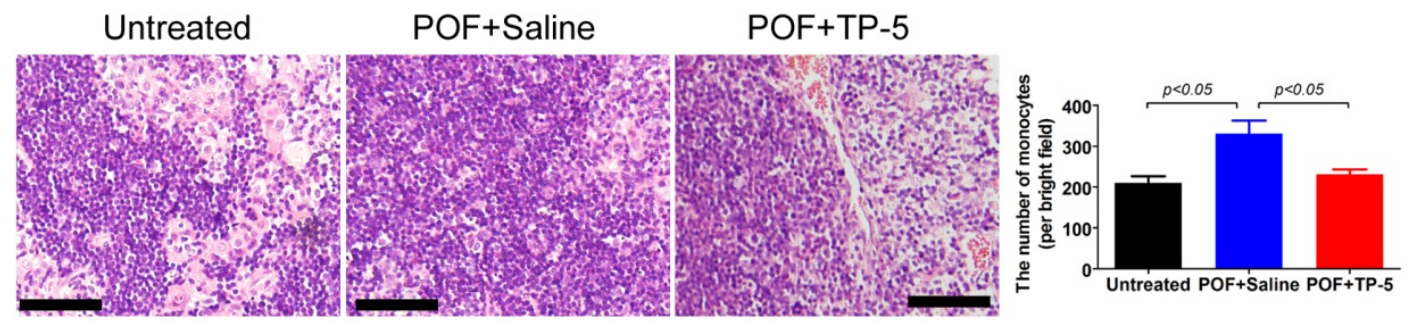

F.

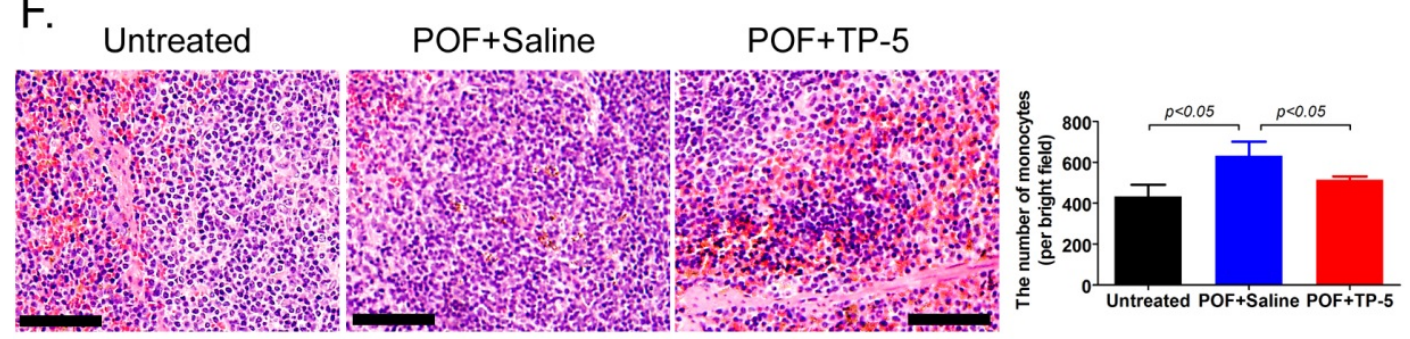

Figure 1. TP-5 significantly improves POF symptoms. (A) H\&E staining of each group of ovarian samples. Black arrow indicates normal follicle, $400 \times$ magnification; blue arrow indicates atretic follicle. Scale bars: $30 \mu \mathrm{m}$. (B) Calculation of atretic follicle proportions. (C) Determination of ovarian weight. (D) ELISA of estrogen levels in peripheral blood. (E) H\&E staining of thymus tissue in each group, $200 \times$ magnification. Scale bars: $30 \mu \mathrm{m}$. (F) H\&E staining of spleen tissue in each group, $200 \times$ magnification. Scale bars: 30 $\mu \mathrm{m}$.

\section{TP-5 significantly improves immune cell proportion and activity}

Flow cytometric analysis of the type and number of immune cells in the ovaries in each group of mice showed that the proportion of helper $\mathrm{T}$ cells $(\mathrm{CD} 3+/ \mathrm{CD} 4+)$ in the ovarian tissue from the TP-5 intervention group was significantly higher than that in the tissues from the control group. In contrast, the proportion of activated $\mathrm{T}$ cells $(\mathrm{CD} 3+/ \mathrm{CD} 8+)$ in the ovarian tissue from the TP-5 intervention group was significantly lower than that in the tissues from the control group (Figure 3A). Simultaneously, the proportion of macrophages (M $\varphi 1)$ cells $(\mathrm{F} 4 / 80+$ / $\mathrm{CD} 68+)$ in the ovarian tissue of the TP-5 intervention group was significantly lower than that in the control group, whereas the proportion of $\mathrm{M} \varphi 2$ cells (F4/80+/
CD206+) was significantly higher than that in the control group (Figure 3B). In addition, the results of qPCR revealed that the expression levles of other inflammatory factor activation biomarkers mRNAs, such as Il1b, Il6, Ifng in CD3+ T cells in POF+TP-5 treated group were significantly lower than those in the control group, exception to Il2 (Figure S1). Meanwhile, the results of qPCR also revealed that the expression levles of other inflammatory factor activation biomarkers mRNAs, such as Il1b, Tnf, Ifng in $\mathrm{F} 4 / 80+$ macrophages $(\mathrm{M} \varphi)$ in POF+TP-5 treated group were significantly lower than those in the control group, exception to differentiation promoting factor Tgfb1 (Figure S1). These experimental results indicated that TP-5 attenuated proportion and activation of CD3+ T cells and type I macrophages. 
A.

SOD

CAT

ATP
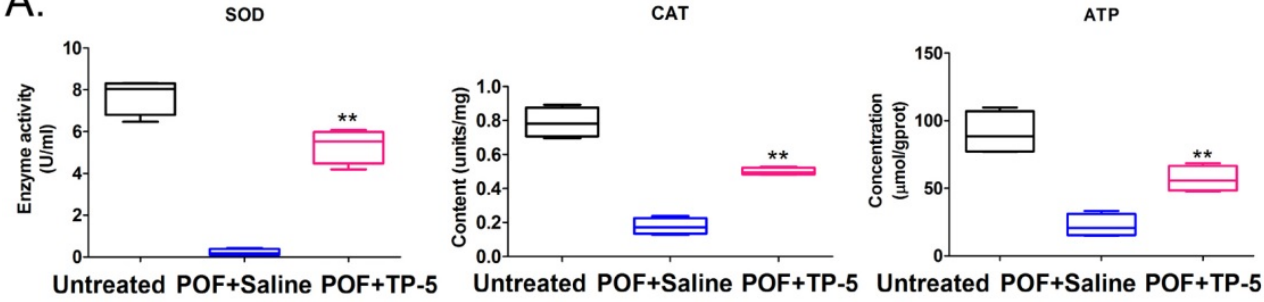

LPO

MDA

Superoxide anion radical
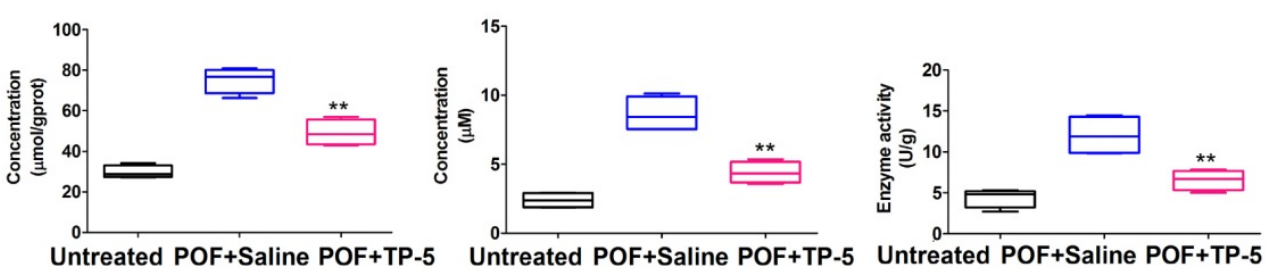

B.
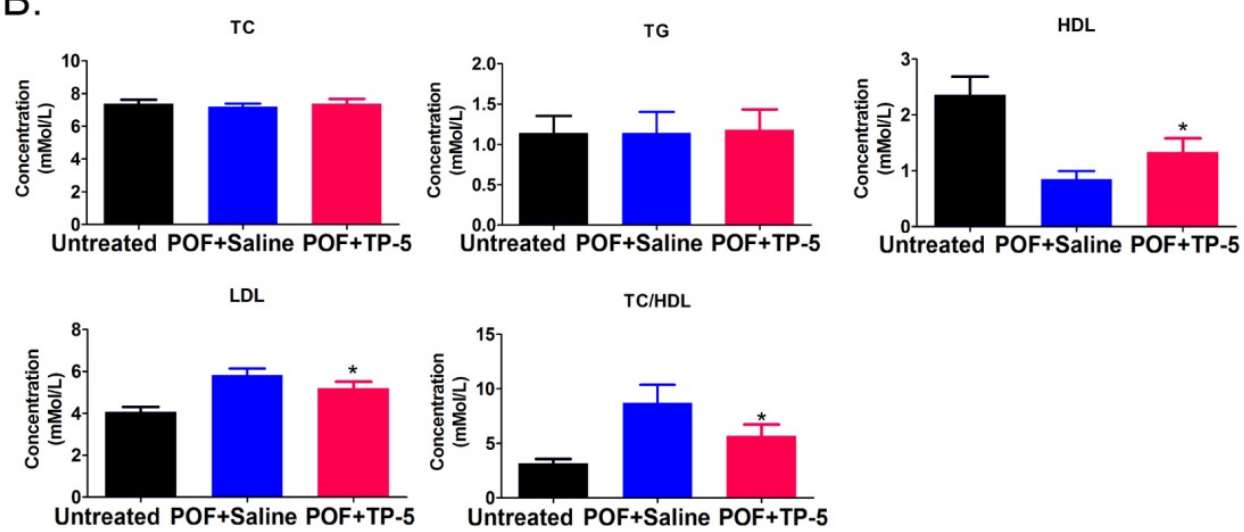

Figure 2. The results of oxidative stress and blood lipid biomarkers. (A) Oxidative stress biomarkers assay. $* * p<0.01$ vs $P O F+S a l i n e ; ~ t$ test; $n=4 . S O D$, Superoxide dismutase; CAT, Catalase; ATP, Adenosine 5'-triphosphate; LPO, Lipid peroxidation; MDA, Malondialdehyde. (B) Blood lipid biomarkers assay. * ${ }^{2}<0.05$ vs POF+Saline; t test; $\mathrm{n}=4$.

\section{TP-5 alters the expression of multiple genes and associated signalling pathways in ovarian tissue}

Ovarian tissue mRNA was collected from a total of six samples in the POF+TP-5, POF+Saline and Untreated groups and sequenced using RNA-Seq transcriptome sequencing. A total of $52.47 \mathrm{~Gb}$ of clean data were obtained. The clean data for each sample reached $8.74 \mathrm{~Gb}$, and the minimum Q30 base percentage was $93.32 \%$. The clean reads for each sample were aligned with the designated reference genome sequence, with efficiency ranging from $95.15 \%$ to $96.63 \%$. RNA-Seq analysis showed differential expression of 14,448 genes among the three groups (Figure 4A, B, C, Table S1). Of these, the POF+TP-5 group had a significant change in mRNA expression levels for a total of 50 genes compared with the $\mathrm{POF}+$ Saline group, of which 43 were significantly upregulated (POF+TP-5/POF+Saline $>10$ and $P<0.01)$ and 17 were significantly downregulated (POF+TP-5/POF+Saline $>0.1$ and $P<$ 0.01). The number of upregulated genes was significantly higher than the number of downregulated genes (Figure 3D, Table S1). Pairwise alignment of the RNA-Seq results revealed that seven genes were differentially expressed in every group.

GO analysis showed that two biological processes, cellular and single-organism processes, contained the genes with the most significant differences in expression between the POF+TP-5 and $\mathrm{POF}+$ Saline groups (Figure $4 \mathrm{E}$ ). Regarding organelle composition, cells and their organelles contained the genes with the most significant differences in expression (Figure 4E). With regard to protein molecular function, binding and catalytic activity pathways contained the functional genes with the most significant differences in expression (Figure 4E). Finally, with respect to the Kyoto Encyclopedia of Genes (KEGG), mTOR signalling pathway contained the genes with the most significant differences in expression (Figure 4F). These experimental results indicated that TP-5 alters the expression of multiple genes and their associated signalling pathways in ovarian tissue. 

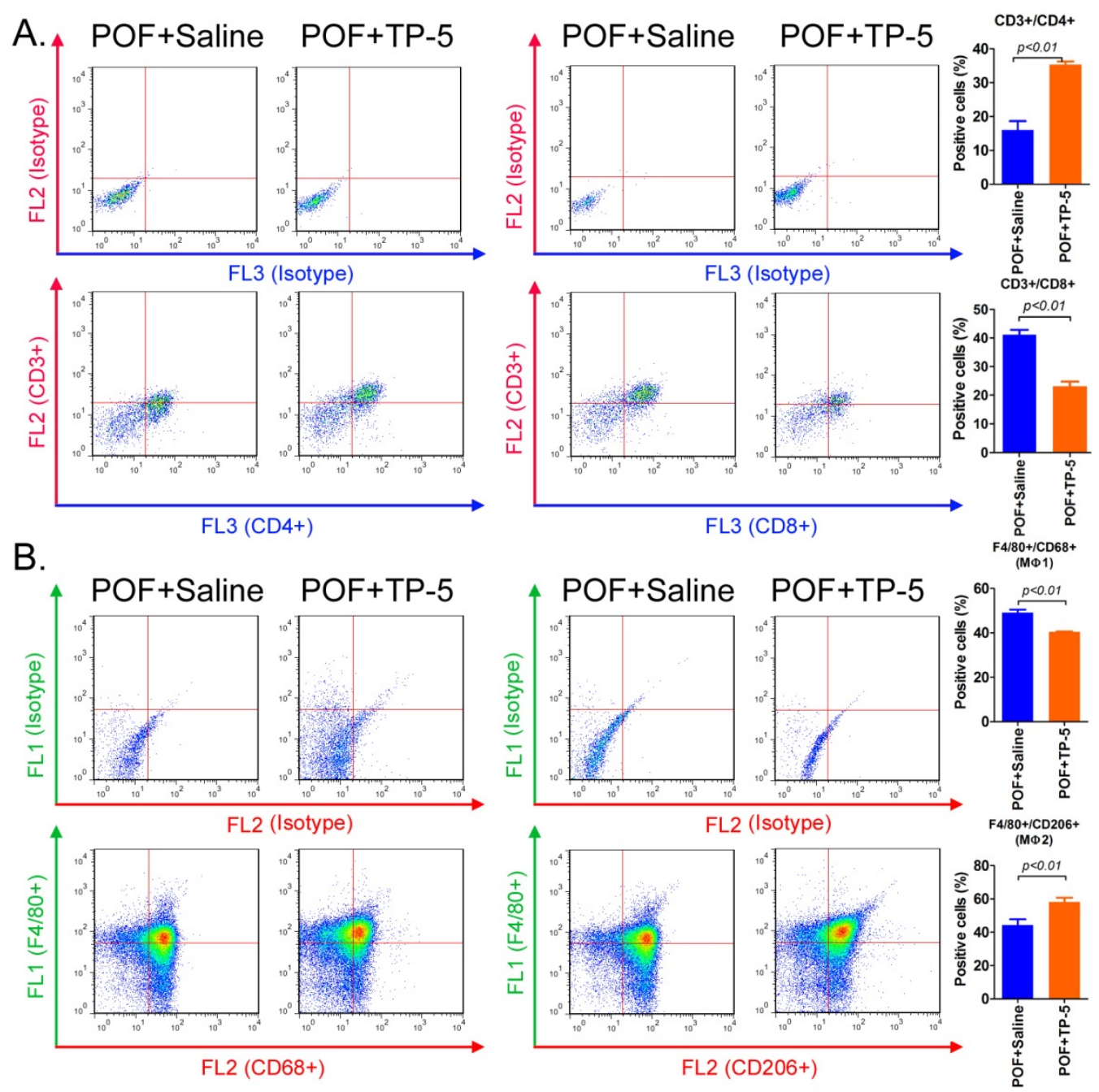

Figure 3. Flow cytometric analysis of immune cell proportions. (A) T lymphocytes. (B) Macrophages.

\section{TP-5 significantly alters the expression level of immunoregulatory factors}

Further analysis of immune system-related data from the RNA-Seq results revealed that the mRNA expression of the interleukin (IL1b, IL6, IL4), NFKB (Nfkbid), and TNF (Tnf, Tnfsf14) families was significantly downregulated in the POF+TP-5 group compared to that in the $\mathrm{POF}+\mathrm{Saline}$ group (Figure $5 \mathrm{~A}$, Table S1), whereas expression of the TGF $\beta$-Smad signaling pathway family (Smad9) was significantly increased (Figure 5A, Table S1). Sequencing results suggested that TP-5 significantly inhibits the expression of interleukin and tumor necrosis factor genes. The results of qPCR were in good agreement with the above RNA-Seq results (Figure 5B). And, the ELISA results showed that IL-1 $\beta$, IL-6, IL-22, TNFa, and $\mathrm{IFN}_{\gamma}$ levels in the peripheral blood of the TP-5 intervention group were significantly lower than those in the POF+Saline group (Figure 5C). Meanwhile, the results of western blot showed that expression levels of NFkB inperipheral blood mononuclear cellnucleuswere significantly downregulated in the POF+TP-5 group compared to them in the POF+Saline group (Figure 5D). These results showed that TP-5 significantly improves the level of immunoregulatory factors.

\section{TP-5 promotes $M \varphi 2$ proportion in the ovary and corrects $P O F$ by regulating the expression of the BMP4/Smad9 signalling pathway}

Immunofluorescence staining showed that the proportions of $\mathrm{F} 4 / 80^{+} / \mathrm{CD}^{206} 6^{+} / \mathrm{BMP}^{+}, \mathrm{F} 4 / 80^{+}$/ $\mathrm{CD}^{206}{ }^{+} / \mathrm{TGF}^{+}, \mathrm{F} 4 / 80^{+} / \mathrm{CD} 8^{+} / \mathrm{Smad}^{+}{ }^{+}$and $\mathrm{F} 4 / 80^{+} / \mathrm{CD} 8^{+} / \mathrm{p}^{-S m a d} 9^{+}$(phosphorylated Smad9) cells in the ovarian tissues of TP-5-intervention mice were significantly lower from those in the control group (Figure 6). And, qPCR results indicated that the expression levels of Tgf $\beta$, Bmp4 and Smad9 in the POF+TP-5 group were significantly lowerthan them in the POF+Saline group (Figure 7A). These results showed that TP-5 promotes the correction of POF by activating the BMP4/Smad9 signalling pathway to promote $\mathrm{M} \varphi 2$ polarization in the ovaries. 

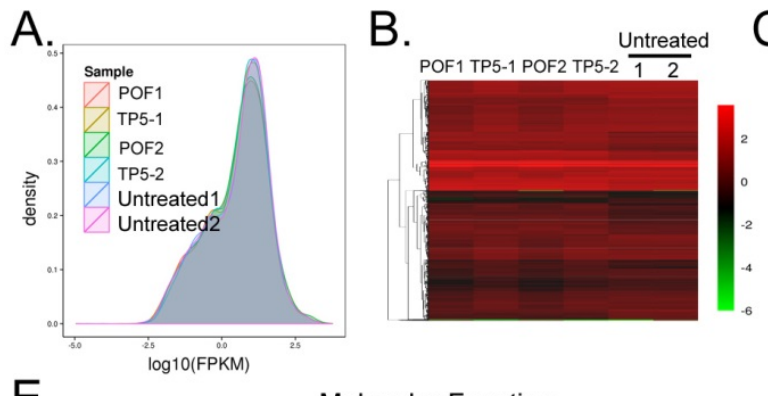

\section{C.}
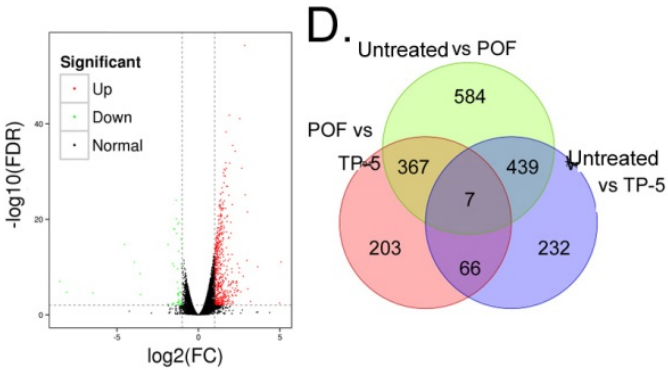

E.
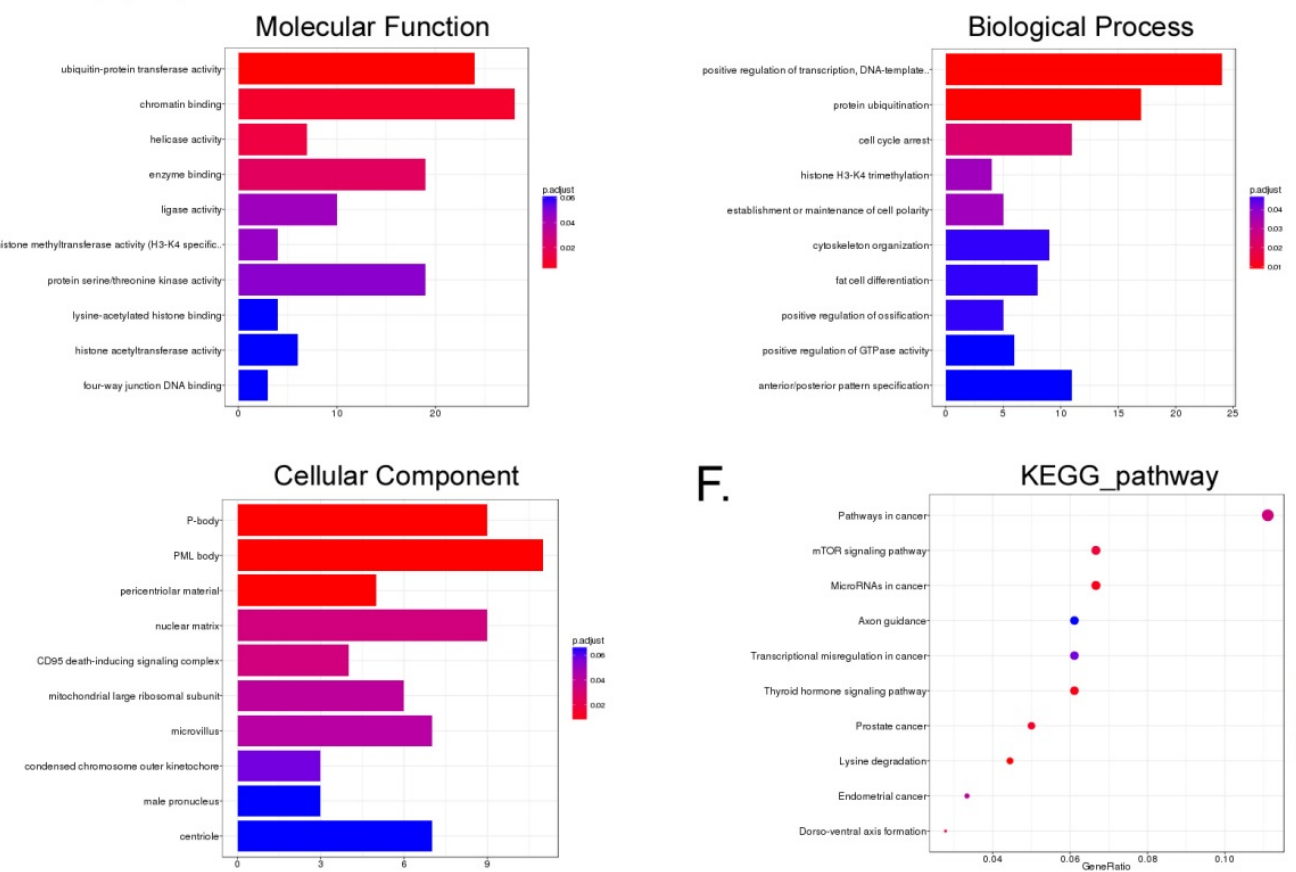

F.

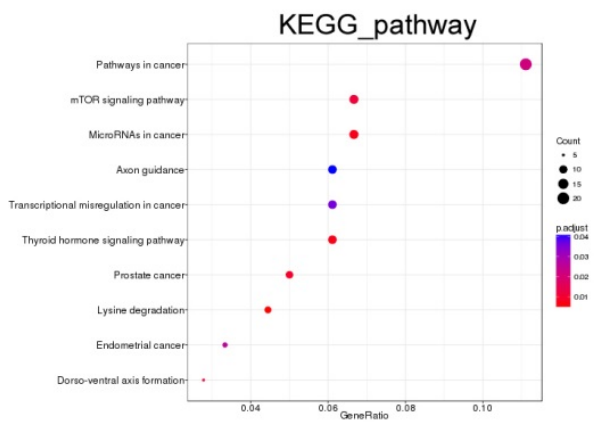

Figure 4. RNA-Seq analysis of gene expression profiles of ovarian tissue in each group. (A) Distribution density of differentially expressed genes in each group. (B) Gene expression heatmap for each group of samples; green and red colors represent downregulated and upregulated expression, respectively. (C) Volcano plot analysis of gene expression for each group of samples; green and red dots represent downregulated and upregulated expression, respectively. (D) Analysis of similarities and differences between gene expression profiles for each group. (E) GO analysis showed that the differences in gene expression between the TP-5 and POF groups were most significant in the cellular process, single-organism process, cell part, binding, and catalytic activity. (F) KEGG analysis showed that genes involved in mTOR signalling pathway.

\section{Discussion}

POF is both a reproductive endocrine disorder and in a broad sense, a form of pathological aging of the reproductive system [1-5]. The pathogenesis of POF involves many factors, and inflammatory activity in the ovary may be one of the main causes of POF in humans [19]. Therefore, targeting the regulation of immune inflammatory responses to a steady state is a rational strategy for POF treatment. On the other hands, a HFHS diet has been recently reported to lead to ovarian dysfunction, which contributes to decreased female reproductive capacity $[8,10-13$, 18].several recent studies have focused on the adverse effects of a HFHS diet on health. Consumption of a HFHS diet can promote the development of various diseases, including cardiovascular disease, tumors, and diabetes via increasing the lipid oxidative stress injury $[7,9,12]$. CD4+ $\mathrm{T}$ cells, $\mathrm{CD} 8+\mathrm{T}$ cells, and macrophages play essential roles in immune responses. T lymphocytes, which are derived from bone marrow-derived lymphoid stem cells, differentiate and mature in the thymus and are distributed to the distant immune organs and tissues via the lymphatic and blood circulation systems to exert their immune functions [20,21]. T cells include subsets, such as helper $\mathrm{T}$ cells (CD4+ T, Th), which support humoral and cellular immunity, and cytotoxic T cells (CD8+ T, Tc), which kill target cells $[20,21]$. Studies have shown lymphocytic infiltration in the ovarian tissues of patients with ovarian insufficiency, suggesting a relationship between immune inflammatory activity and ovarian function $[19,22]$. Mø are white blood cells derived from monocytes and located in the tissues. Both Mø and monocytes are phagocytic cells involved in non-specific defense (innate immunity) and specific defense (cellular immunity) in vertebrates. The primary function of $\mathrm{Mø}$ is to phagocytose and digest cell debris and pathogens as immotile or motile cells, and to activate the response of lymphocytes and other immune cells to pathogens [23]. Wu et al. indicated 
that although the interaction between ovarian steroid hormones and pituitary gonadotropins is a major regulator of the ovarian cycle, there is significant evidence indicating that Mø act as critical support cells for optimal fertility due to their nutritional function in reproductive tissues. The specific localization and distribution of Mø in the ovary at different stages of the menstrual cycle and their presence in human follicular fluid during ovulation suggest that macrophages play different roles in the ovarian functions, including follicular development and tissue reorganization during ovulation, and corpus luteum formation, degeneration [23-25]. Thus, $\mathrm{T}$ cells and Mø are closely related to ovarian function.

A growing number of studies have pointed out that cytokines, especially inflammatory factors, can induce ovarian tissue or follicle aging and death
[26-29]. Paul F Terranova et al reported that TNFa induced serum amyloid A 3 among acute-phase proteins in mouse granulosa cells by activating NF-kBsignaling via p55 TNFa receptor type 1 [28]. Meanwhile, Jodi Anne Flaws also reported that TNF and both receptors (TNFRSF1A and TNFRSF1B) are expressed in neonatal mouse ovaries and that TNF promotes oocyte death in neonatal ovaries in vitro [29]. Their results and other studies also indicated that TNFa has been shown directly to be a trigger of ovarian aging at the level of primordial follicles [28, 29]. Our research found that the concentrations of some inflammatory factors (IL-1 $\beta, \mathrm{TNFa}$, etc.) in the peripheral blood mononuclear cell of POF mice group were elevated significantly. According to above reports, we have reason to believe that HFHS promoted ovarian aging through inflammatory

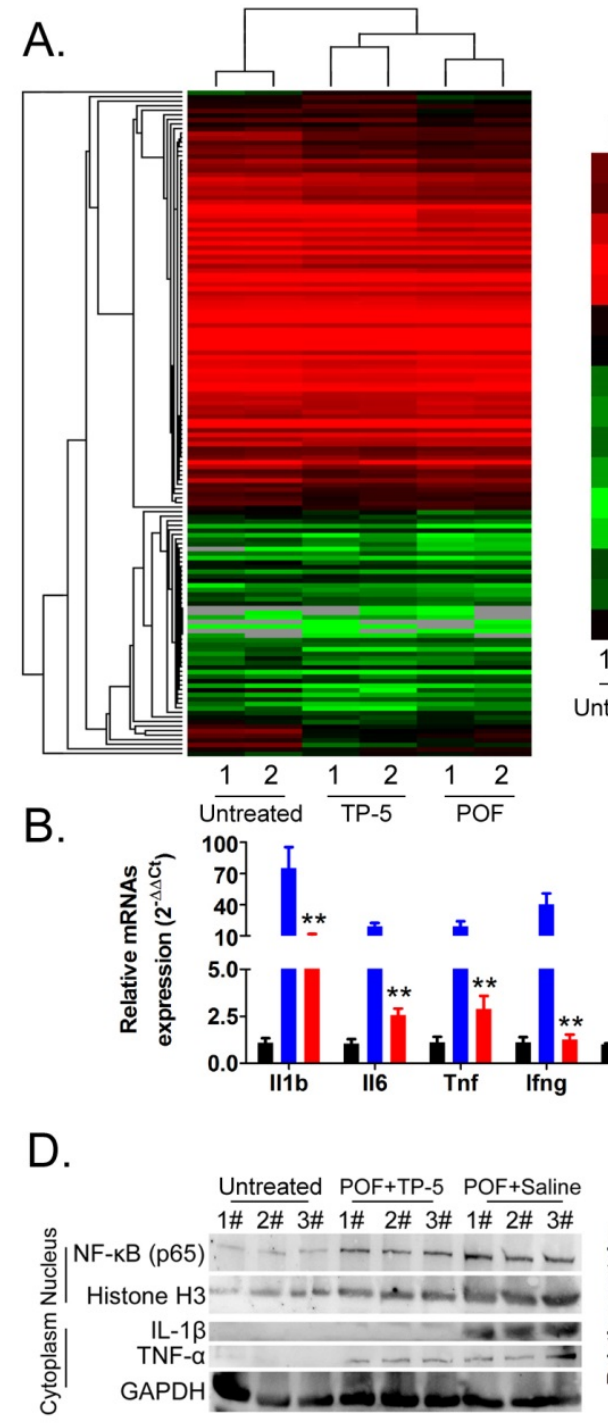

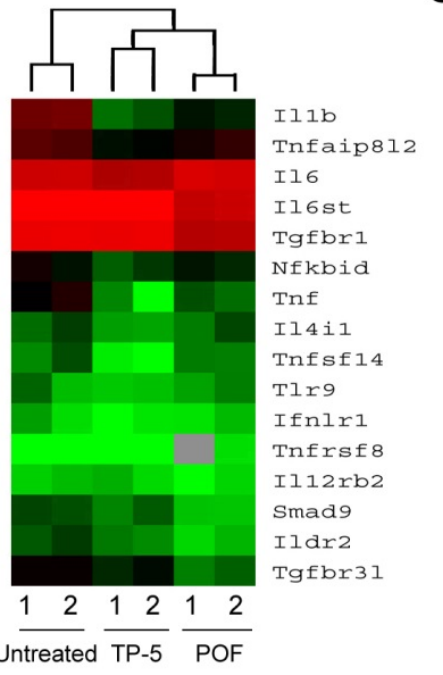

$\begin{array}{lllll}-5 & -2.5 & 0 & 2.5 & 5\end{array}$
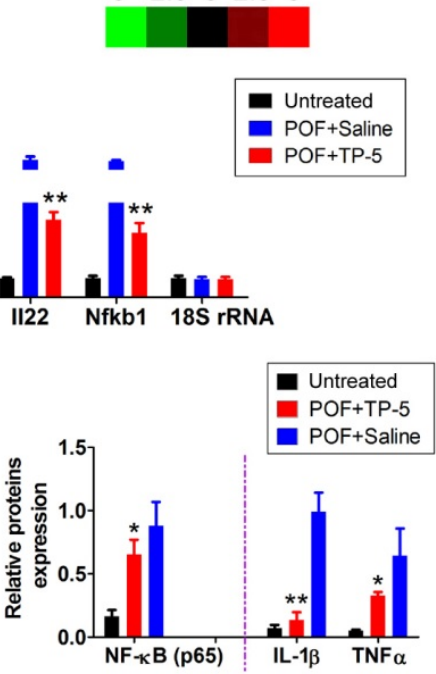

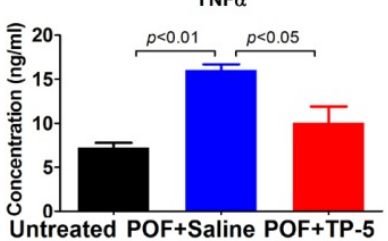

C.

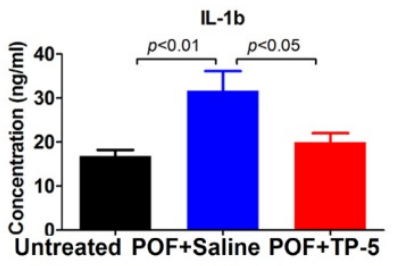

IL-6

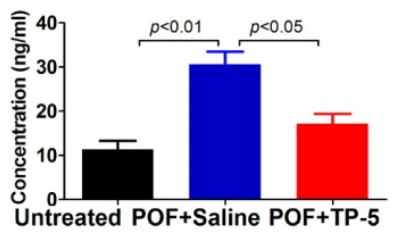

IL-22

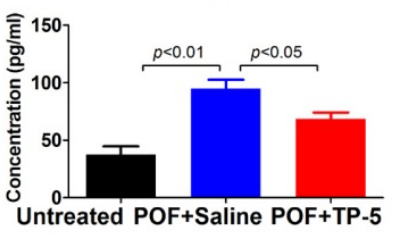

Untreated POF+Saline POF+TP-5

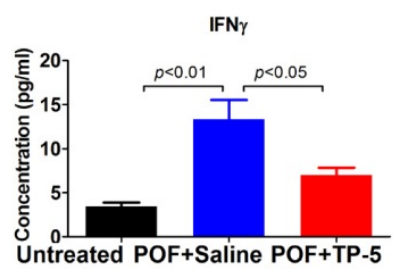

Figure 5.RNA-Seq analysis of differentially expressed genes of immunoregulatory factors. (A) Heatmap analysis of differential expression of genes in the interleukin, NFKB, and TNF families; green and red colors represent downregulated and upregulated expression, respectively. (B) qPCR results. ** $<<0.01$ vs POF+Saline; $t$ test; $\mathrm{n}=4$. (C) ELISA results for interleukin, TNFa, and IFNYin the peripheral blood of mice in each group. (D) Western blotting assay results. * $\mathrm{p}<0.05$ vs POF+Saline; ** $\mathrm{p}<0.01$ vs POF+Saline; $t$ test; $n=3$. 
factors, especially IL-1 $\beta$, TNFa and so on. In addition, some studies have reported that a HFHS diet increases the risk of obesity, tumor formation, and cardiovascular disease [7-9] and adversely affects ovarian function and ovum quality [10-12]. The HFHS diet significantly induced abnormal glucose and lipid metabolism, increased oxidative free radicals and accelerated cell aging [10-12]. Meanwhile, The oxidative stress reaction derived to the HFHS diet lead to over activation of immune cells and long-term release of inflammatory factors, and eventually lead to immune imbalance [10-12]. However, some studies reported that TP-5 had therapeutic effects on autoimmune diseases such as rheumatoid arthritis, type II diabetes, and female climacteric syndrome [14-18]. Since the HFHS diet and TP-5 have effects by regulating immune activity, we have reason to believe that TP-5 could also treat POF derived to the HFHS diet [10-12]. When mice were treated to TP-5, the phenotype of premature ovarian failure had significantly alleviated, and at the same time, the concentration of inflammatory factors in peripheral blood mononuclear cell decreased significantly. It can be seen that the concentration of inflammatory factors and the phenotype of POF were weakened by TP- 5 at the same time. Therefore, we suggested that TP-5 alleviated the pathological aging of ovary by reducing the inflammatory factors.According to some reports, TP-5 effectively regulates immune cell activity and homeostasis and the release of immune factors; however, there are no reports on its efficacy in the treatment of $\mathrm{POF}$, and it is unclear whether it can alleviate the symptoms of POF by targeting the regulation of T cells and Mø. One of the roles of TP-5 is to induce $\mathrm{T}$ cell differentiation by selectively inducing the conversion of Thy-1-prethymocytes into Thy-1+ T cells [14-18], a process mediated by elevated intracellular cAMP levels. TP-5 also enhances the phagocytic function of macrophages, enhances the enzymatic activity and phagocytosis of polymorphonuclear neutrophils, increases the number of circulating antibodies, and augments the immune function of red blood cells [14-18]. In our study, we found that TP-5 significantly reduced the proportions of activated $\mathrm{T}$ cells $(\mathrm{CD} 3+/ \mathrm{CD} 8+)$ and $\mathrm{M} \varphi 1 \quad(\mathrm{~F} 4 / 80+/ \mathrm{CD} 68+)$ and the expression of inflammatory factors in POF mice decreased with the number of inflammatory cells.

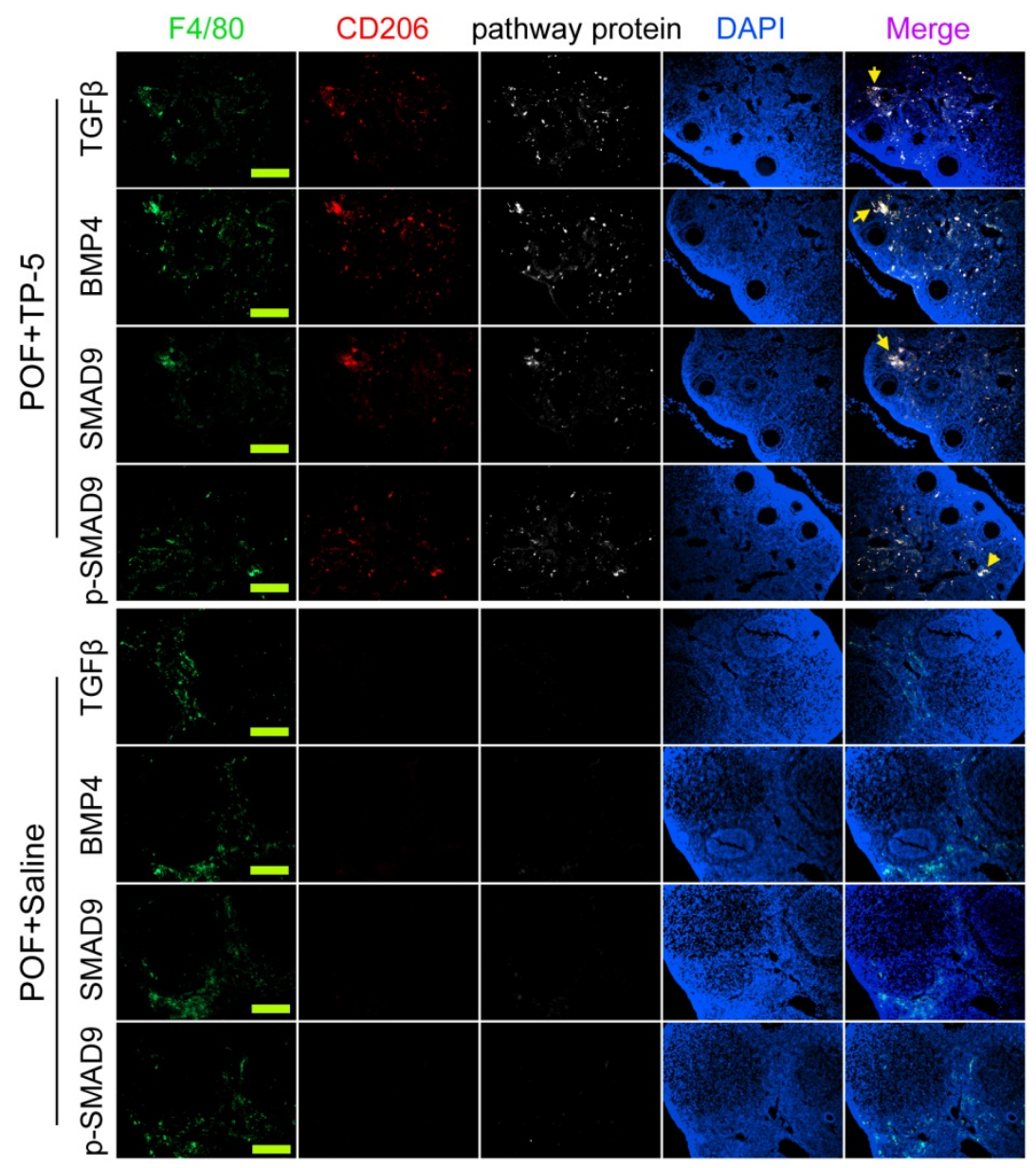

Figure 6. Immunofluorescence staining of key proteins of the BMP4/Smad9 signaling pathway of Mø2 cells in ovarian tissue. The pathway protein refers to TGF $\beta$, BMP4, SMAD9, p-SMAD9 (phosphorylated SMAD9). Scale bars: $30 \mu \mathrm{m}$. 


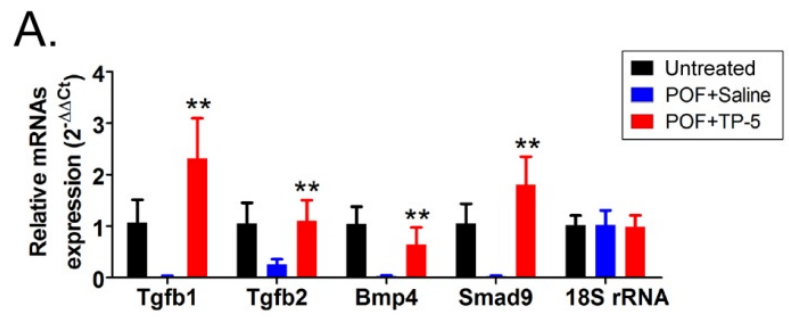

B.

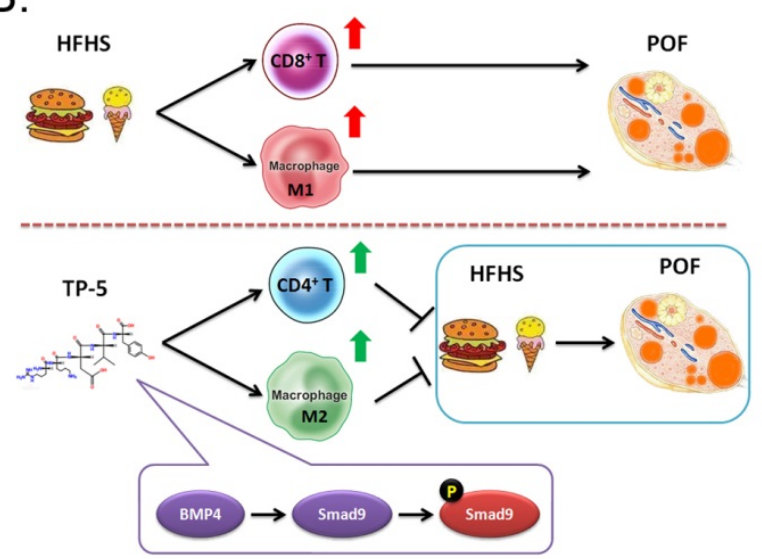

Figure 7. TP-5 treatment of murine POF via activation of the BMP4/ Smad9 signalling pathway. (A) qPCR results. ${ }^{* *} \mathrm{p}<0.01$ vs POF+Saline; $t$ test; $n=4$. (B) The mechanism underlying TP-5 treatment of murine POF via activation of the BMP4/Smad9 signalling pathway.

Finally, in light of the evidence showing that TP-5 modulated Mø proportion and activity $(\mathrm{Mø1} \rightarrow \mathrm{M} \varnothing 2)$, we investigated the molecular biological mechanism of this process. We found overexpression of BMP4/Smad9 signalling pathway molecules in the ovarian Mø2 cells in the TP-5 group. TGF- $\beta$ superfamily signalling plays an important role in the regulation of cell growth, differentiation, and development in numerous biological systems. In general, signal transduction is initiated by ligandinduced oligomerization of the serine/threonine receptor kinases and phosphorylation of cytoplasmic signal transduction molecules, such Smad2 and Smad3 in the TGF- $\beta$ /activin pathway, or Smad1/5/9 in the bone morphogenetic protein (BMP) pathway. Phosphorylation of the carboxyl-terminus of Smads by the activated receptor results in binding to the common signal transduction factor, Smad4, and translocation into the nucleus. Binding of activated Smads to transcription factors results in cell-specific transcriptional regulation of various biological effects [30-33]. Therefore, we hypothesize that TP-5 converts Mø1 into Mø2 in POF ovaries by activating the BMP4/Smad9 signalling pathway in ovarian Mø cells (Figure 7B).

In summary, TP-5 attenuated activation and proportion of immune cells, and weakens the release of inflammatory factors and lipid oxidative stress injury by reducing BMP4/Smad9 signalling in the ovaries of POF mice, thereby improving ovarian tissue function.

\section{Supplementary Material}

Supplementary figures and tables. http://www.medsci.org/v18p3544s1.pdf

\section{Acknowledgements}

This work was supported by grant from the National Natural Science Foundation of China (No. 81973899); and shanghai training project for leading talents of traditional Chinese medicine (ZY(2018-2020)-RCPY-1024).

In advance of submission, we had this manuscript copyedited by a professional English editing service that specializes in scientific papers.

\section{Ethical approval}

All the animal experiments were conducted in accordance with the guidelines of the NIH for the care and use of laboratory animals and with the 1964 Helsinki declaration. The study protocol was also approved by the Committee on the Use of Live Animals in Teaching and Research, Shanghai Geriatric Institute of Chinese Medicine, Shanghai, China. This article does not contain any studies with human participants performed by any of the authors.

\section{Competing Interests}

The authors have declared that no competing interest exists.

\section{References}

1. Liu T, Huang Y, Guo L, Cheng W, Zou G. CD44+/CD105+ human amniotic fluid mesenchymal stem cells survive and proliferate in the ovary long-term in a mouse model of chemotherapy-induced premature ovarian failure. Int J Med Sci. 2012; 9: 592-602

2. Xiong Y, Liu T, Wang S, Chi H, Chen C, Zheng J. Cyclophosphamide promotes the proliferation inhibition of mouse ovarian granulosa cells and premature ovarian failure by activating the lncRNA-Meg3-p53-p66Shc pathway. Gene. 2017; 596: $1-8$

3. Ai A, Xiong Y, Wu B, Lin J, Huang Y, Cao Y, et al. Induction of miR-15a expression by tripterygium glycosides caused premature ovarian failure by suppressing the Hippo-YAP/TAZ signaling effector Lats1. Gene. 2018; 678: 155-63.

4. Liu T, Wang S, Li Q, Huang Y, Chen C, Zheng J. Telocytes as potential targets in a cyclophosphamide-induced animal model of premature ovarian failure. Mol Med Rep. 2016; 14: 2415-22.

5. Liu T, Huang Y, Zhang J, Qin W, Chi H, Chen J, et al. Transplantation of human menstrual blood stem cells to treat premature ovarian failure in mouse model. Stem Cells Dev. 2014; 23: 1548-57.

6. Liu T, Liu Y, Huang Y, Chen J, Yu Z, Chen C, et al. miR-15b induces premature ovarian failure in mice via inhibition of alpha-Klotho expression in ovarian granulosa cells. Free radical biology \& medicine. 2019; 141: 383-92.

7. Goncalves MD, Lu C, Tutnauer J, Hartman TE, Hwang SK, Murphy CJ, et al. High-fructose corn syrup enhances intestinal tumor growth in mice. Science. 2019; 363: 1345-9.

8. Tappy L, Le KA. Metabolic effects of fructose and the worldwide increase in obesity. Physiol Rev. 2010; 90: 23-46.

9. Hodge AM, Bassett JK, Milne RL, English DR, Giles GG. Consumption of sugar-sweetened and artificially sweetened soft drinks and risk of obesity-related cancers. Public Health Nutr. 2018; 21: 1618-26.

10. Wang N, Luo LL, Xu JJ, Xu MY, Zhang XM, Zhou XL, et al. Obesity accelerates ovarian follicle development and follicle loss in rats. Metabolism: clinical and experimental. 2014; 63: 94-103. 
11. Wu Y, Zhang Z, Liao X, Wang Z. High fat diet triggers cell cycle arrest and excessive apoptosis of granulosa cells during the follicular development. Biochemical and biophysical research communications. 2015; 466: 599-605.

12. Liu G, Shi F, Blas-Machado U, Duong Q, Davis VL, Foster WG, et al. Ovarian effects of a high lactose diet in the female rat. Reproduction, nutrition, development. 2005; 45: 185-92.

13. Liu T, Lin J, Chen C, Nie X, Dou F, Chen J, et al. MicroRNA-146b-5p overexpression attenuates premature ovarian failure in mice by inhibiting the Dab2ip/Ask1/p38-Mapk pathway and gammaH2A.X phosphorylation. Cell Prolif. 2021; 54: e12954.

14. Li J, Cheng $\mathrm{Y}$, Zhang $\mathrm{X}$, Zheng $\mathrm{L}$, Han $\mathrm{Z}$, Li $\mathrm{P}$, et al. The in vivo immunomodulatory and synergistic anti-tumor activity of thymosin alpha1-thymopentin fusion peptide and its binding to TLR2. Cancer Lett. 2013; 337: 237-47.

15. Liu Z, Zheng $X$, Wang J, Wang E. Molecular analysis of thymopentin binding to HLA-DR molecules. PLoS One. 2007; 2: e1348.

16. Fan YZ, Chang H, Yu Y, Liu J, Zhao L, Yang DJ, et al. Thymopentin (TP5), an immunomodulatory peptide, suppresses proliferation and induces differentiation in HL-60 cells. Biochim Biophys Acta. 2006; 1763: 1059-66.

17. Xiaojing C, Yanfang L, Yanqing G, Fangfang C. Thymopentin improves cardiac function in older patients with chronic heart failure. Anatol J Cardiol. 2017; 17: 24-30.

18. Wu C, Zhang M, Zhang Z, Wan KW, Ahmed W, Phoenix DA, et al. Thymopentin nanoparticles engineered with high loading efficiency, improved pharmacokinetic properties, and enhanced immunostimulating effect using soybean phospholipid and PHBHHx polymer. Mol Pharm. 2014; 11: 3371-7.

19. Silva CA, Yamakami LY, Aikawa NE, Araujo DB, Carvalho JF, Bonfa E. Autoimmune primary ovarian insufficiency. Autoimmun Rev. 2014; 13: 427-30.

20. Farhood B, Najafi M, Mortezaee K. CD8(+) cytotoxic T lymphocytes in cancer immunotherapy: A review. J Cell Physiol. 2019; 234: 8509-21.

21. Nguyen QP, Deng TZ, Witherden DA, Goldrath AW. Origins of CD4(+) circulating and tissue-resident memory T-cells. Immunology. 2019; 157: 3-12.

22. Yan G, Schoenfeld D, Penney C, Hurxthal K, Taylor AE, Faustman D. Identification of premature ovarian failure patients with underlying autoimmunity. J Womens Health Gend Based Med. 2000; 9: 275-87.

23. Wu R, Van der Hoek KH, Ryan NK, Norman RJ, Robker RL. Macrophage contributions to ovarian function. Hum Reprod Update. 2004; 10: 119-33.

24. Norman RJ, Brannstrom M. White cells and the ovary--incidental invaders or essential effectors? J Endocrinol. 1994; 140: 333-6.

25. Cohen PE, Nishimura K, Zhu L, Pollard JW. Macrophages: important accessory cells for reproductive function. J Leukoc Biol. 1999; 66: 765-72.

26. He H, Xu P, Zhang X, Liao M, Dong Q, Cong T, et al. Aging-induced IL27Ra signaling impairs hematopoietic stem cells. Blood. 2020; 136: 183-98.

27. Kleefeldt F, Bommel H, Broede B, Thomsen M, Pfeiffer V, Worsdorfer P, et al. Aging-related carcinoembryonic antigen-related cell adhesion molecule 1 signaling promotes vascular dysfunction. Aging Cell. 2019; 18: e13025.

28. Choi H, Ignacio RMC, Lee ES, Roby KF, Terranova PF, Son DS. Localization of Serum Amyloid A3 in the Mouse Ovary. Immune Netw. 2017; 17: 261-8.

29. Greenfeld CR, Roby KF, Pepling ME, Babus JK, Terranova PF, Flaws JA. Tumor necrosis factor (TNF) receptor type 2 is an important mediator of TNF alpha function in the mouse ovary. Biol Reprod. 2007; 76: 224-31.

30. Horbelt D, Denkis A, Knaus P. A portrait of Transforming Growth Factor beta superfamily signalling: Background matters. Int J Biochem Cell Biol. 2012; 44: 469-74.

31. Ikushima H, Miyazono K. TGFbeta signalling: a complex web in cancer progression. Nat Rev Cancer. 2010; 10: 415-24.

32. Sapkota G, Alarcon C, Spagnoli FM, Brivanlou AH, Massague J. Balancing BMP signaling through integrated inputs into the Smad1 linker. Mol Cell. 2007; 25: 441-54.

33. Alarcon C, Zaromytidou AI, Xi Q, Gao S, Yu J, Fujisawa S, et al. Nuclear CDKs drive Smad transcriptional activation and turnover in BMP and TGF-beta pathways. Cell. 2009; 139: 757-69. 